\title{
EL EXPERIMENTO NATURAL COMO UN NUEVO DISEÑO CUASI-EXPERIMENTAL EN INVESTIGACION SOCIAL Y DE SALUD.
}

\author{
NATURAL EXPERIMENT AS A NEW QUASI-XPERIMENTAL DESIGN IN \\ SOCIAL AND HEALTH-RELATED SCIENCES.
}

\author{
LILIAN NASS KUNSTMANN*, JOSÉ MANUEL MERINO E.**
}

\begin{abstract}
RESUMEN
En las ciencias sociales y de la salud actualmente comienza a ser conocido un diseño denominado como "experimento natural", que se caracteriza por ser un tipo de cuasi-experimento en que la asignación de tratamiento no es hecha por el investigador sino por un agente exógeno tal como una ley, una política sectorial o un fenómeno natural. Esa intervención exógena implícita permite que se formen grupos con bajos niveles de sesgos de selección inicial, por lo que la comparación de resultados encontrados en esos grupos (grupos afectados por la ley, la política o fenómeno natural respecto del grupo contrafáctico que no es afectado) permite predecir los efectos de la ley, política sectorial o fenómeno natural casi tan eficientemente como en la creación de grupos aleatorios de tratamiento y control.
\end{abstract}

Palabras claves: experimento natural, diseño metodológico, sesgos de selección

\begin{abstract}
Currently in social science research it is used a new research design defined as a "natural experiment", where the researcher has none participation in the treatment assignment because it has been directly done for an exogenous agent such as a law, a social policy or a natural event. This implicit exogenous intervention allows that low-level pre-determined selection bias groups be formed. Therefore, the comparison on results being found between these groups (law-affected group, social policy-affected group, or natural event-affected group versus contrafactic groups not being affected) allow to predict the effect of this law, social policy, or natural event almost so efficiently like happen in randomly created treatment-and-control groups.
\end{abstract}

Key words: natural experiment, design research, selection bias

Fecha Recepción: 18 diciembre 2007 Fecha Aceptación: 21 octubre 2008

\section{INTRODUCCION}

El experimento natural corresponde a la aplicación de una estrategia de investigación nueva en ciencias sociales y de la salud. Este diseño o modelo metodológico es un tipo de "cuasi-experimento" (Trochim, 2001), que se caracteriza porque el investigador no interviene en la creación de los grupos de tratamiento y control. Este rol asignador de los sujetos de investigación a los grupos de tratamiento y control es efectuado por un agente exógeno. Ese agente exógeno no es el investigador, más generalmente puede ser un fenómeno natural, una ley o un cambio de ley (Jacobsen, J.; Pearce, J.W. \& Rosenbloom, J. 1999; Hotz, J.; McElroy, S. \& Sanders, S. 2005; New, 2006). El investigador aprovecha para fines de estudio la creación de esos grupos cuasi-aleatorios de comparación: uno de intervención, tratamiento o exposición (esto es, con la ley; o con el cambio de ley; o con el fenómeno natural), el otro de control o de comparación (sin efectos de la ley, del cambio de ley o del fenómeno natural).

*Cirujano dentista, Magíster en Salud Familiar, Profesor Asociado Departamento de Odontología Restauradora, Facultad de Odontología, Universidad de Concepción, Concepción, Chile. Email: lnass@udec.cl

${ }^{* *} \mathrm{PhD}$ Sociology, Profesor Titular Departamento de Sociología, Facultad de Ciencias Sociales, Universidad de Concepción, Concepción, Chile. Email: jmerino@udec.cl 
En economía y otras ciencias sociales se viene últimamente aplicando con gran frecuencia los llamados experimentos naturales. Estos estudios examinan los resultados de observaciones en grupos intervenidos y los comparan con grupos que no han estado expuestos al tratamiento. En estos estudios existe variación exógena en las variables que determinan la asignación de la intervención. Generalmente son cambios políticos (Dynarski, 1999) u otros eventos administrativos los que permiten al investigador obtener variación exógena en las principales variables explicativas (Barceinas, F.; Oliver, J.; Raymond, J. \& Roig, J. 2000). Este fenómeno es especialmente útil en situaciones donde las estimaciones son sesgadas debido a variación endógena provenientes de variables omitidas o sesgos de selección. Estas estrategias están siendo usadas muy extensamente para analizar un amplio rango de problemas (Garruto, R.M.; Little, G.D.; James, D. \& Brown, D. 1999; O'Connor, 2003; Petticrew et al., 2005). Otras situaciones favorables para ser utilizarlas son los cambios en las políticas de gobierno, que frecuentemente son aplicadas en algunos grupos de la población y no en otros (Duflo, 2002).

La escasez y limitaciones de los experimentos sociales han sido por largo tiempo identificadas como las mayores dificultades en el progreso de las ciencias sociales y de la salud. Rosenzweig y Wolpin (2000) enumeran cinco estudios que emergieron desde mecanismos biológicos y climáticos (nacimiento de mellizos, nacimiento de gemelos, fecha de nacimiento, género y eventos climáticos) que han permitido la aplicación de experimentos naturales. Estos eventos han sido usados para estudiar los retornos de la escolaridad y la experiencia laboral en el mercado, el consumo, ahorro y aporte laboral en los cambios temporales o permanentes en la renta y participación femenina en el campo laboral y cambios en la fertilidad. La ventaja del experimento natural es que la suposición de exogeneidad de las variables instrumentales empleadas es más creíble que aquellas usadas en casi todos los otros estudios (Duncan, G.; Magnuson, K. \& Ludwig, J. 2004.; Jaramillo, 2005).

Otros investigadores los han aplicado en el ámbito de la familia (Chevalier, 2004; Evans \& Lien 2005; Lalive \& Zweimuller, 2005) y en contextos extra-familiares (Bluedorn \& Cascio, 2005; Dunning, 2007). Rutter (1998), compara los efectos de la privación, abandono y desapego, sufridos en distintos grados por niños abandonados en orfelinatos, sobre el desarrollo psicológico posterior. GoldinMeadow (1997) estudió niños sordos cuyos padres los expusieron al lenguaje convencional de señas a temprana edad, respecto de otros niños de la misma condición que no son expuestos a dicho entrenamiento o que lo son tardíamente en la vida, para entender los efectos de la formación temprana del lenguaje en niños con déficit auditivo severo. Duncan et al., (2004) también menciona el estudio de Bronars y Geogger (1994), que investiga mellizos nacidos de madres solteras que son separados a temprana edad y criados en ambientes culturales diferentes como una fuente de variación exógena que explica resultados diferentes de las experiencias de vida en niños genéticamente iguales, aunque expuestos a ambientes sociales distintos.

En el campo de la salud este tipo de estudios sustituyen al experimento aleatorio en situaciones en que esta estrategia no es aplicable, siendo un aporte a la investigación en el área (Sekikawa et al., 2003). Un ejemplo local de aplicación de esta metodología es un estudio donde se compara la calidad de la atención odontológica en los Centros de Salud Familiar (CESFAM) y Centros de Salud (CES) para analizar la influencia sobre los usuarios de la introducción del modelo de atención con enfoque de salud familiar que comienza a ser aplicado en los centros de salud del país (Nass, 2006). Los grupos de pacientes fueron conformados de acuerdo a una estrategia inicial de pareamiento simple que consideró características socio-demográficas, socio-económicas y socio-contextuales, por 
lo que debe considerarse que las muestras de pacientes de CES y CESFAM, en este estudio, no son diferentes estadísticamente en edad, estado civil, escolaridad, ocupación, ingresos y número de hijos. La única diferencia es que los CESFAM aplican un paradigma predominantemente familiar en la atención de salud, situación que es contrafáctica respecto de los CES que se caracterizan por una atención predominantemente individual. La utilización del experimento natural es plenamente apropiada, porque existen dos grupos a contrastar, uno de intervención y otro de comparación, exógenamente creados mediante la implementación (o no) del modelo nuevo de atención en salud.

\section{DISCUSION}

Mediante el diseño de experimento natural, ambos grupos, el de tratamiento y el de control, deben ser iguales en la mayoría de sus características observadas iniciales, lo que puede ser ratificado técnicamente mediante un procedimiento de emparejamiento (matching) simple o multidimensional. Por lo tanto las diferencias en los resultados en relación a las variables estudiadas, deberían ser explicadas fundamentalmente por la exposición a la intervención exógena en el grupo tratamiento, exposición ausente en el grupo control (Angrist, J.; Bettinger, E.; Bloom, E.; King, E. \& Kramer, M. 2002) (Conniffe, D; Gash, V. \& O'Connell, Ph. 2000) (Shin-Yi, C.; Jin-Tan, L.; Grossman, M. \& Joyce, T. 2007). La ventaja del diseño de experimento natural es que la suposición de aleatoriedad de las variables instrumentales empleadas es más creíble que aquellas usadas en casi todos los otros estudios. Es importante considerar que la dirección del sesgo es difícil de predecir ex-ante. Muchos estudios no-experimentales y observacionales, en general, están predispuestos a tener sesgos endógenos y aunque el potencial de sesgo es a menudo apuntado como una limitación, los investigadores y con- sumidores de investigación tienen poca idea de la magnitud, dirección y efectos negativos que tiene sobre los resultados de la investigación (Meyer, 1994).

Se recomienda a los investigadores utilizar la ventaja de la asignación cuasi-aleatoria que proviene del experimento natural, puesto que produce variación exógena en fenómenos del ámbito de la salud, en la familia y variables contextuales de interés. Este diseño metodológico genera entonces menores problemas de validez interna que los estudios observacionales tradicionales, ya que no se selecciona a los individuos, el ambiente es creado por una medida externa, no existiendo sesgo. Los grupos que se crean por la aplicación de políticas, reformas, eventos naturales, pueden legítima y creativamente ser comparados en términos de resultados específicos, aislando efectivamente el efecto de esa aplicación como un mecanismo de inferencia causal válida debido a la naturaleza exógena y, por tanto, insesgada, de la creación de los grupos que se comparan.

\section{REFERENCIAS}

Angrist, J.; Bettinger, E.; Bloom, E.; King, E. \& Kramer, M. (2002). Vouchers for private schooling in Colombia: Evidence from a randomized natural experiment. The American Economic Review 92(5), 1535-1558.

Barceinas, F.; Oliver, J.; Raymond, J. \& Roig, J. (2000). Rendimientos Públicos de la educación y restricción presupuestaria. Papeles de Economía Española, 86, 236-248.

Bluedorn, J. \& Cascio, E. (2005). Education and intergenerational mobility: Evidence from a natural experiment in Puerto Rico. Nutfield College Economic Paper WP 21; pp. 1-66.

Bronars, S. \& Groegger, J. (1994). The economic consequences of unwed motherhood: Using twin births as a natural experiment. American Economic Review, 84, 1141-1156.

Conniffe, D; Gash, V. \& O'Connell, Ph. (2000). Evaluating states programs: Natural experiments and propensity scores. The Economic and Social Review 31(4), 283-308. 
Chevalier, A. (2004). Parent education and child education: A natural experiment. IZA Discussion Paper 1153, 1- 47.

Duflo, E. (2002). Empirical Methods. MIT 14.771/ Harvard 2390b. Disponible en: http://www. Jourdan.ens.fr/piketty/fichiers/enseig/ecoineg/ articl/Duflo2002.pdf (consultado el 4 de mayo de 2005).

Duncan, G.; Magnuson, K. \& Ludwig, J. (2004). The endogeneity problem in development studies. Research in Human Development (1-2), 59-80.

Dunning, T. (2007). Improving causal inference: Strenghts and limitations of natural experiments. Political Research Quarterly. Print version forthcoming. Online version available at http:/intl-prq.sagepub.com/pap. $\mathrm{dtl}$ as of October 3.

Dynarski, S. (2003). Does aid matter?. Measuring the effect of students aid on college attendance and completion. American Economic Review 93(1), 279-288.

Evans, W. \& Lien, D. (2005). The benefits of prenatal care: evidence from the PAT bus strike. Journal of Econometrics. 125 (1), 207-239.

Garruto, R.M.; Little, G.D.; James, D. \& Brown, D. (1999). Natural experiments models: The global search for biomedical paradigms among traditional, modernizing, and modern populations. Proceedings of the National Academy of Sciences of the United States of America 96(18), 10536-10543.

Goldin-Meadow, S. (1997). When gestures and words speak differently. Current Directions in Psychological Science, 6, 138-143.

Hotz, J.; McElroy, S. \& Sanders, S. (2005). Teenage childbearing and its life cycle consequences: Exploiting a natural experiment. The Journal of Human Resources 40 (3), 683-715.

Jacobsen, J.; Pearce, J.W. \& Rosenbloom, J. (1999). The effects of childbearing on married women's labor supply and earnings: Using twins births as a natural experiment. The Journal of Human Resources 34 (3), 449-474.

Jaramillo, M. (2005). Evaluación experimental del programa Formación de Líderes Empresariales en elDepartamento deHuancavélica. Disponible en: http://www.grade.org.pe/download/docs/ CIDHuancavelica.pdf (consultado el 1 de junio de 2005).
Lalive, R. \& Zweimüller, J. (2005) Does parental leave affect fertility and return-to-work? Evidence from a true natural experiment. IZA Discussion Paper Series \# 1613, 1- 44.

Meyer, B. (1995). Natural and quasi-experiments experiments in economics. Journal of Business \& Economic Statistics, Vol. 13, No. 2, 151161.

Nass, L. (2006). Estudio comparativo de la calidad de la atención odontológica en Centros de Salud y Centros de Salud Familiar de la Octava Región durante el año 2005: Un experimento natural. Tesis no publicada. Programa de Magister en Salud Familiar, Facultad de Medicina, Escuela de Graduados; Universidad de Concepción, Chile.

New, M. (2006). Using natural experiments to analyze the impact of state legislation on the incidence of abortion. Working Paper The Heritage Foundation CDA06-01, 1- 9.

O'Connor, T. (2003). Natural experiment to study the effects of early experience: progress and expectations. Developments and Psychopatology 15, 837-852.

Petticrew, M.; Cummins, S.; Ferrel, C.; Findlay, A.; Higgins, C.; Hoy, C.; Kearns, A. \& Sparks, L. (2005). Natural experiments: An underused tool for public health? Public Health 119, 751-757.

Rosenzweig, M. \& Wolpin, K. (2000). Natural "natural experiments" in economics. Journal of Economic Literature. 38, 827-874.

Rutter, M. (1998). Developmental catch-up, and deficit, following adoption after severe global early privation. Journal of Child Psychology \& Psychiatry \& Allied Disciplines. 39, 465-476.

Sekikawa, A.; Horiuchi, B.; Edmundowicz, D.; Ueshima, H.; Curb, J.; Sutton-Tyrrel, K.; Okamura, T.; Kadowaki, T.; Kashiwagi, A.; Mitsunami, K.; Murata, K.; Nakamura, Y.; Rodriguez, B. \& Kuller, L. (2003). A natural experiment in cardiovascular epidemiology in the early $21^{\text {st }}$ century. Hearth. 89(3), 255257.

Shin-Yi, C.; Jin-Tan, L.; Grossman, M. \& Joyce, T. (2007). Parental education and child health: Evidence from a natural experiment in Taiwan. National Bureau of Economic Research Working Paper 13446, 1-48.

Trochim, W. (2001). The Research Methods Knowledge Base. Cincinnatti, OH: Atomic Dog Publishing. 